\title{
Different enteral nutrition formulas have no effect on glucose homeostasis but on diet-induced thermogenesis in critically ill medical patients: a randomized controlled trial
}

\author{
Marlene Wewalka $^{1} \cdot$ Andreas Drolz $^{1} \cdot$ Berit Seeland $^{1} \cdot$ Mathias Schneeweiss $^{1} \cdot$ Monika Schmid $^{1}$. \\ Bruno Schneeweiss ${ }^{2} \cdot$ Christian Zauner ${ }^{1}$
}

Received: 1 October 2017 / Revised: 12 January 2018 / Accepted: 14 January 2018

(c) Macmillan Publishers Limited, part of Springer Nature 2018

\begin{abstract}
Background/Objectives Hyperglycemia is common in critically ill patients and associated with increased mortality. It has been suggested that different nutrition formulas may beneficially influence glucose levels in surgical intensive care patients. In this prospective randomized clinical cohort study we investigated glucose homeostasis in response to different enteral nutrition formulas in medical critically ill patients.

Subjects/Methods 60 medical critically ill patients were randomized to receive continuous fat-based (group A, $n=30$ ) or glucose-based enteral nutrition (group $\mathrm{B}, n=30$ ) for seven days. Indirect calorimetry was performed to determine energy demand at baseline and on days 3 and 7. Glucose levels and area under the curve (AUC), insulin demand, glucose variability, and calorie and substrate intake per $24 \mathrm{~h}$ were assessed for 7 days.

Results Over the course of 7 days patients had similar average daily glucose $(p=0.655)$, glucose AUC (A: 758 (641-829) $\mathrm{mg} / \mathrm{d} \mathrm{l} / \mathrm{day}$ vs B: $780(733-845) \mathrm{mg} / \mathrm{dl} / \mathrm{day}, p=0.283)$, similar overall insulin demand (A: 153.5 (45.3-281.5) IE vs B: 167.9 (82.3-283.8) IE, $p=0.525)$, and received similar amounts of enteral nutrition per $24 \mathrm{~h}$. Resting energy expenditure was similar at baseline (A: $1556(1227-1808) \mathrm{kcal} /$ day vs B: 1563 (1306-1789) kcal/day, $p=0.882)$ but energy expenditure increased substantially over time in group A $(p<0.0001)$, but not in group B $(p=0.097)$.

Conclusion Fat-based and glucose-based EN influence glucose homeostasis and insulin demand similarly, yet diet-induced thermogenesis was substantially higher in critically ill patients receiving fat-based enteral nutrition.
\end{abstract}

\section{Introduction}

Nutritional support has become an integral part of therapy in critically ill patients in order to minimize negative effects of starvation as well as energy and protein catabolism [1,2]. It has been shown that adequate caloric intake decreases severe complications and improves clinical outcome yet both underfeeding and overfeeding should be avoided [3, 4]. To this point the enteral route is the preferred way of

Marlene Wewalka

marlene.wewalka@meduniwien.ac.at

1 Medical University of Vienna, Department of Internal Medicine III, Division of Gastroenterology and Hepatology, Intensive Care Unit, Waehringer Guertel 18-20, 1090 Vienna, Austria

2 Department of Internal Medicine, LKH Kirchdorf, Hausmanningerstrasse 8, 4560 Kirchdorf, Austria feeding, since it is assumed that enteral feeding is associated with structural and functional integrity of the intestine, thereby preventing increased gut permeability, which is associated with bacterial translocation [5]. This might be related to a lower incidence of infections [1]. Nevertheless, enteral feeding can cause gastrointestinal intolerance possibly leading to unintentional underfeeding.

Hyperglycemia related to stress is a common finding in critically ill patients and is associated with poor outcome. It is caused by a complex interplay between counterregulatory hormones and cytokines leading to increased hepatic glucose production and peripheral insulin resistance $[6,7]$. This stress-induced endogenous hyperglycemia can be aggravated by an exogenous glucose supply during artificial nutrition [6]. Thus, decreasing the amount of glucose provided exogenously could mitigate hyperglycemia. This has been shown previously in 16 trauma patients, where the administration of a total parenteral nutrition (TPN) with a decreased fraction of glucose induced lower 
serum glucose and insulin concentrations compared to a glucose-based TPN [8]. A decreased serum glucose concentration was also confirmed in trauma patients during combined enteral and parenteral nutrition with a decreased glucose content [9]. However, the nutritional regimen in the latter study did not conform to nutritional guidelines $[1,2]$. In addition, the two groups received different fat emulsions [9].

Alterations in glucose homeostasis have not yet been investigated in critically ill medical patients during continuous enteral nutrition (EN). We have therefore undertaken this prospective, randomized clinical cohort study to evaluate glucose homeostasis in critically ill medical patients receiving either fat-based or glucose-based EN continuously over a study period of seven days. The primary outcome of the study was daily average glucose, secondary outcome measures were insulin demand per $24 \mathrm{~h}$, glucose variability and nutrition related side effects.

\section{Patients and Methods}

\section{Subjects}

Critically ill medical patients admitted to our medical intensive care unit (ICU) were eligible for the study if they needed nutritional support, were mechanically ventilated with fraction of inspired oxygen $\left(\mathrm{FiO}_{2}\right) \leq 60 \%$, and were older than 18 years. Exclusion criteria that prohibited from participation were contraindications against nutritional therapy such as hemodynamic shock (lactate of $\geq 4 \mathrm{mmol} / \mathrm{l}$ ) or severe hypertriglyceridemia $(\geq 450 \mathrm{mg} / \mathrm{dl}$ ) or contraindications against $\mathrm{EN}$ in particular, like intestinal obstruction, disruption or severe bleeding of upper gastrointestinal tract. Furthermore, patients with a diagnosis of diabetes were excluded from the study.

Over the course of two years, sixty consecutive critically ill medical patients were included into the present study and were randomized $1: 1$ by a physician not involved in the study using randomization in permuted blocks, in order to receive either fat-based (Diben, Fresenius Kabi, Austria; group A, $n=30$ ) or glucose-based (Fresubin original fiber, Fresenius Kabi, Austria, group B, $n=30$ ) EN continuously for seven days. Nutritional support was started within $72 \mathrm{~h}$ after ICU admission. Patients undergoing therapeutic hypothermia after cardiac arrest were assessed and enrolled after rewarming.

For each patient age, sex, reason for ICU admission, comorbid conditions, body mass index (BMI), severity of illness, assessed by the Simplified Acute Physiology Score (SAPS) II [10] and the Sequential Organ Failure Assessment (SOFA) [11] Score at ICU admission were documented. Blood glucose levels were assessed at least three times per day, drawn from an arterial line. Additional measurements were performed to the treating physician's discretion. Insulin demand and calorie intake from EN as well as other energy sources per $24 \mathrm{~h}$ were documented. Hyperglycemia was defined as blood glucose concentrations $>150 \mathrm{mg} / \mathrm{dl}$. Hypoglycemia was defined as blood glucose levels $<60 \mathrm{mg} / \mathrm{dl}$, whereas severe hypoglycemia was defined as a blood glucose concentration $<40 \mathrm{mg} / \mathrm{dl}$. In addition, we assessed nutrition related side effects like diarrhea, vomiting, increased gastric residual volume (GRV) (i.e., $>500 \mathrm{ml}$ per day) [12], hypertriglyceridemia (serum triglycerides $>350 \mathrm{mg} / \mathrm{dl}$ ) and cholestasis (defined by alkaline phosphatase $>180 \mathrm{U} / \mathrm{l}$ and gamma-GT of $>240 \mathrm{U} / \mathrm{l})$. If patients started to eat spontaneously, they had to terminate the study early, as calorie and substrate intake could not be accurately determined in case of oral food intake.

The ethics committee of the Medical University of Vienna reviewed and approved the study. Patients were consented in accordance with the Austrian legislation. The study was performed in accordance with the ethical standards defined in the revised Helsinki Declaration.

\section{Enteral Nutrition}

Both fat-based and glucose-based EN are approved formulas that are used in the routine treatment. Fat-based EN formulas contain $45 \%$ fat, $37 \%$ carbohydrates, $18 \%$ protein, and $2.3 \mathrm{~g}$ of fiber per $100 \mathrm{ml}$, whereas glucose-based EN formulas are comprised of $30 \%$ fat, $55 \%$ carbohydrates, $15 \%$ protein, and contain $1.5 \mathrm{~g}$ of fiber per $100 \mathrm{ml}$. Both formulas have a caloric density of $1 \mathrm{kcal} / \mathrm{ml}$ and contain rape seed oil and sunflower oil. Initial assessment of resting energy expenditure (REE) was performed for each patient using the technique of indirect calorimetry. Target energy was $25 \%$ above the measured REE [13]. Both study groups received early EN that was initiated with the target dosage and continuously administered at a constant rate for 7 days via a nasogastric tube.

\section{Indirect Calorimetry}

To determine REE indirect calorimetry was performed in a fasting state over an overnight fast of $8-9 \mathrm{~h}$ at baseline. Indirect calorimetry was repeated to assess energy expenditure (EE) on days 3 and 7 during continuous EN. Measurements were done under stable conditions; in case of changes in ventilator settings, pain or agitation, the study was delayed. Furthermore, we did not perform indirect calorimetry in patients with need for hemodialysis [14]. Respiratory gas exchange was measured by a computerized open-circuit indirect calorimetry (Deltatrac Metabolic Monitor II, Datex Instruments, Helsinki, Finland) for 
Table 1 Patient Characteristics $(n=60)$

\begin{tabular}{lllr}
\hline Characteristic & Group A & Group B & $p$-value \\
\hline Sex (m/f) & $12 / 18$ & $16 / 14$ & 0.438 \\
Age (years) & $60 \pm 12$ & $58 \pm 16$ & 0.657 \\
BMI $\left(\mathrm{kg} / \mathrm{m}^{2}\right)$ & $26.2 \pm 5.2$ & $27.5 \pm 4.4$ & 0.294 \\
SAPS II & $58 \pm 14$ & $63 \pm 13$ & 0.147 \\
SOFA & $10 \pm 3$ & $11 \pm 4$ & 0.525 \\
Reason for ICU admission & & & \\
CPR & 13 & 11 & 0.793 \\
Respiratory insufficiency & 9 & 7 & 0.771 \\
Sepsis & 2 & 6 & 0.254 \\
Cardiogenic shock & 1 & 0 & $>0.99$ \\
Coma/status epilepticus & 4 & 5 & $>0.99$ \\
Other & 1 & 1 & $>0.99$ \\
\hline
\end{tabular}

$\chi^{2}$-test or Fisher's exact test, as appropriate

$B M I$ body mass index, $C P R$ cardiopulmonary resuscitation, SAPS II Simplified Acute Physiology Score II, SOFA sequential organ failure assessment

30-60 min until stable conditions with maximum changes of $\pm 5 \%$ were achieved [15]. The gas analyzers were calibrated before each measurement using a calibration gas of known composition (QuickCal Calibration Gas, Datex, Helsinki, Finland). Oxygen consumption $\left(\mathrm{VO}_{2}\right)$ and carbon dioxide production $\left(\mathrm{VCO}_{2}\right)$ were calculated according to the Haldane equation [16]. Measurements were performed every minute and the results of the last 20 min of each measurement were averaged. From the rates of $\mathrm{VO}_{2}$ and $\mathrm{VCO}_{2}$, the respiratory quotient (RQ) and REE or EE are calculated and output by the Deltatrac Metabolic Monitor II [17, 18]. Non-protein RQ was determined by subtracting protein oxidation from total gas exchange [19].

\section{Further Calculations}

Daily glucose variability was calculated as daily SD of glucose $\times 100 /$ daily average glucose ( $\Delta$ glucose) [20]. Glucose area under the curve (AUC) was calculated from average daily blood glucose over the entire study period.

\section{Statistical Analysis}

Statistical analysis was performed using SPSS 19.0 (IBM Corp., Chicago, IL). GraphPad PRISM 6 (GraphPad Software, La Jolla, CA) was used to create figures. Patient characteristics are presented as mean $\pm \mathrm{SD}$, results as median and 25-75\% interquartile range (IQR). Unpaired Student's $T$-test was used for group comparisons for normally distributed variables and Mann-Whitney $U$-test for skewed data. For categorical variables $\chi^{2}$-test or Fisher's exact test were used as appropriate. Friedman test was calculated to assess changes over time within one group. Repeated measures ANOVA was performed to evaluate differences between groups over time. Results with a $p$-value of $\leq 0.05$ were considered statistically significant. All participants that were enrolled in the study entered the statistical analyses. Sample size calculation was based on a previous study by Tappy et al [8].

\section{Results}

\section{Patient Characteristics}

A total of 60 critically ill medical patients admitted to a medical ICU were randomized to receive either a fat-based (group A, $n=30$ ) or a glucose-based (group B, $n=30$ ) EN. The patient characteristics are summarized in Table 1 . Gender distribution, age, BMI, severity of illness as assessed by SAPS II and SOFA scores, and reason for ICU admission did not differ between groups.

Study duration was $6.0 \pm 1.7$ days in group A and $5.9 \pm$ 2.1 days in group B $(p=0.792)$, yielding 178 patient days and 177 patient days, respectively $(p=1.0)$. In both groups, $73.3 \%$ completed the entire study duration of seven days ( $p$ $=1.0$ ). Of the patients who terminated the study early in group A, $23.3 \%$ died and $3.3 \%$ no longer needed EN, whereas in group B 20\% died and $6.7 \%$ no longer needed $\mathrm{EN}(p=1.0)$. At baseline, $73.3 \%$ of patients in group A and $60 \%$ of patients in group B received vasopressor therapy ( $p$ $=0.412$ ). Norepinephrine was the primary vasoactive agent in both groups with an average dosage of $9.8 \pm 15.2 \mathrm{mg} /$ day in group A and $5.9 \pm 9.4 \mathrm{mg} /$ day in group B, respectively ( $p$ $=0.242$ ). ICU mortality was found to be $43 \%$ in group A and $30 \%$ in group B $(p=0.422)$.

\section{Metabolic Studies}

At baseline, indirect calorimetry, performed after an overnight fast, revealed that $\mathrm{VO}_{2}, \mathrm{VCO}_{2}, \mathrm{REE}, \mathrm{RQ}$, and nonprotein RQ did not differ between the two groups (Fig. 1, Table 2).

Over the entire study duration EE significantly increased in the group receiving fat-based EN, whereas it remained unchanged in the patients receiving glucose-based EN (Fig. 1, Table 2).

On days 3 and 7, during continuous EN, patients had similar $\mathrm{VO}_{2}, \mathrm{VCO}_{2}$, EE, RQ, and non-protein RQ (Table 2).

\section{Energy and Substrate Intake}

Patients received similar amounts of EN each day throughout the study period and over the entire study period (group A: 79 (64.3-88.3)\%, group B: 83.3 (72.5-89.5)\%; $p$ $=0.315$; Fig. 2a). Overall caloric intake including EN, 


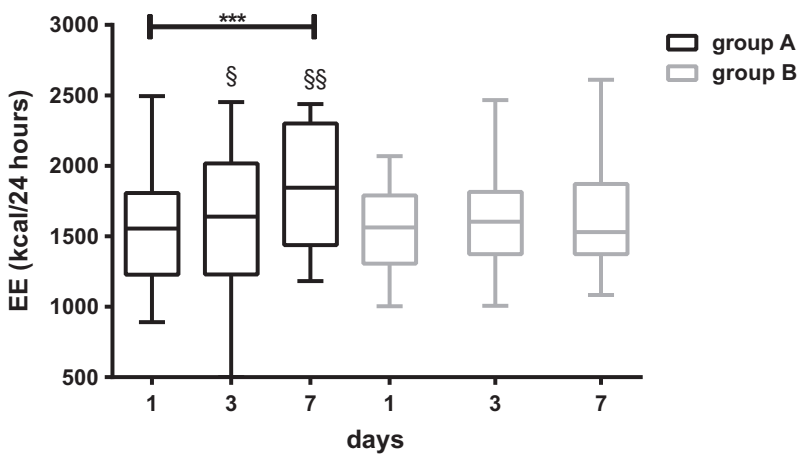

Fig. 1 Energy expenditure on days 1, 3, and 7. Measurement on day 1 after an overnight fast, measurements on days 3 and 7 under continuous EN. $* * *<0.001$ day 1 vs. day $7 . \S<0.05$ vs. day $1, \S \S<0.01$ vs. day 1. Black-fat-based enteral nutrition (group A). Gray-glucose-based enteral nutrition (group B)

glucose administration, and propofol trended to be higher in group B on day 2 (group A: 1487 (1028-1832) kcal/day, 1697 (1396-1980) kcal/day; $p=0.066$ ), but did not differ between groups on the other study days or over the entire study period (Fig. 2b).

Patients in group A, who were fed with a fat-based formula, received $44 \%$ more fat $(419$ (269-497) g, 329 $(250-386) \mathrm{g} ; p=0.01)$ as well as $51 \%$ less carbohydrates (694 (418-850) g, 1059 (782-1321) g; $p=0.029$ ) over the entire course of the study compared to patients of group B. Protein supply did not differ between both groups (group A: 377 (232-447)g, group B: $367(279-431) \mathrm{g} ; p=0.745)$ throughout the study.

\section{Measures of Glucose Homeostasis}

Fasting plasma glucose (FPG) was elevated but similar in both groups (group A: 128 (110-170) mg/dl, group B: 123 (98-153) $\mathrm{mg} / \mathrm{dl} ; p=0.570$ ). Average daily blood glucose concentration was higher in group A on day 1 (group A: 143 (130-156) mg/dl, group B: 137 (121-147) $\mathrm{mg} / \mathrm{dl} ; p=$ 0.048 ) but did not differ any day thereafter as well as over the entire study period ( $p=0.655$ ) (Fig. 2c). Hyperglycemic event rate (group A: $n=200 / 927$ (21.6\%), group B: $n=$ 207/934 (22.2\%); $p=0.718$ ), glucose AUC (group A: 758 (641-829) $\mathrm{mg} / \mathrm{dl} / \mathrm{day}$, group B: 780 (733-845) mg/dl/day; $p=0.283$ ) and $\Delta$ glucose (Fig. 2d) were comparable between the groups. Daily insulin demand per $24 \mathrm{~h}$ and total insulin demand over the course of the whole study period (group A: 153.5 (45.3-281.5) IE, group B: 167.9 (82.3-283.8) IE; $p=0.525)$ were similar between groups (Fig. 2e). During the study period we observed very low rates of hypoglycemic events (group A: $n=2 / 927$; group B: $n=1 / 934$ hypoglycemic episodes, $p=1.0$ ), however, both events in group A were episodes of severe hypoglycemia.

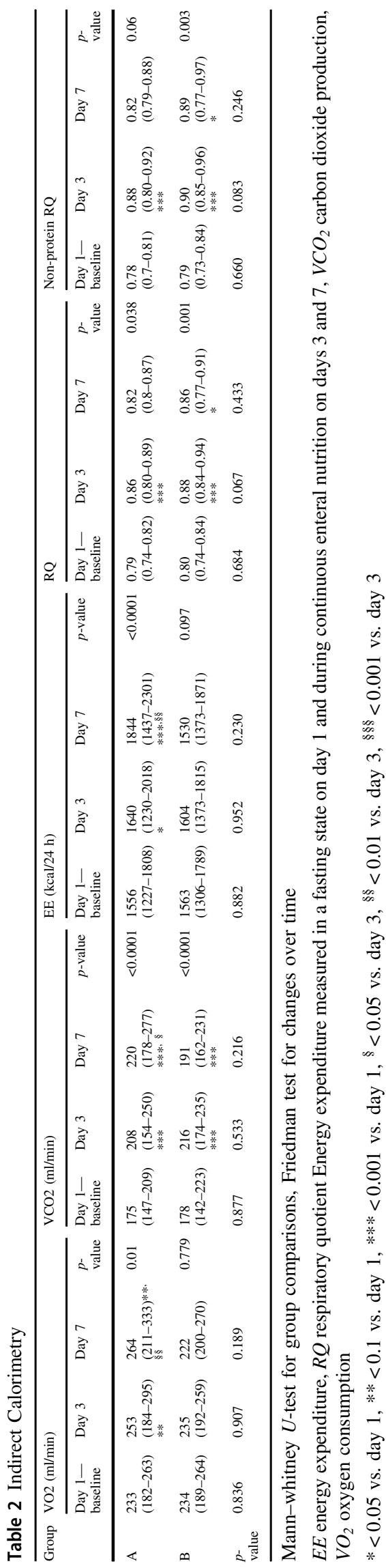


a

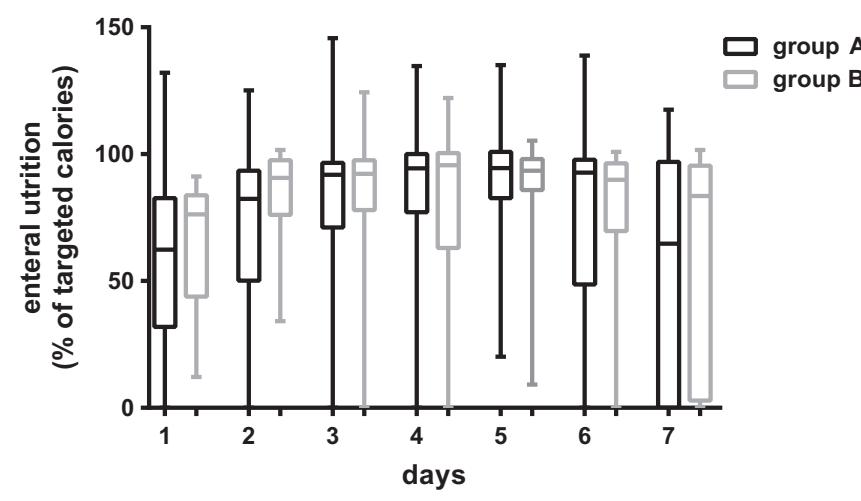

C
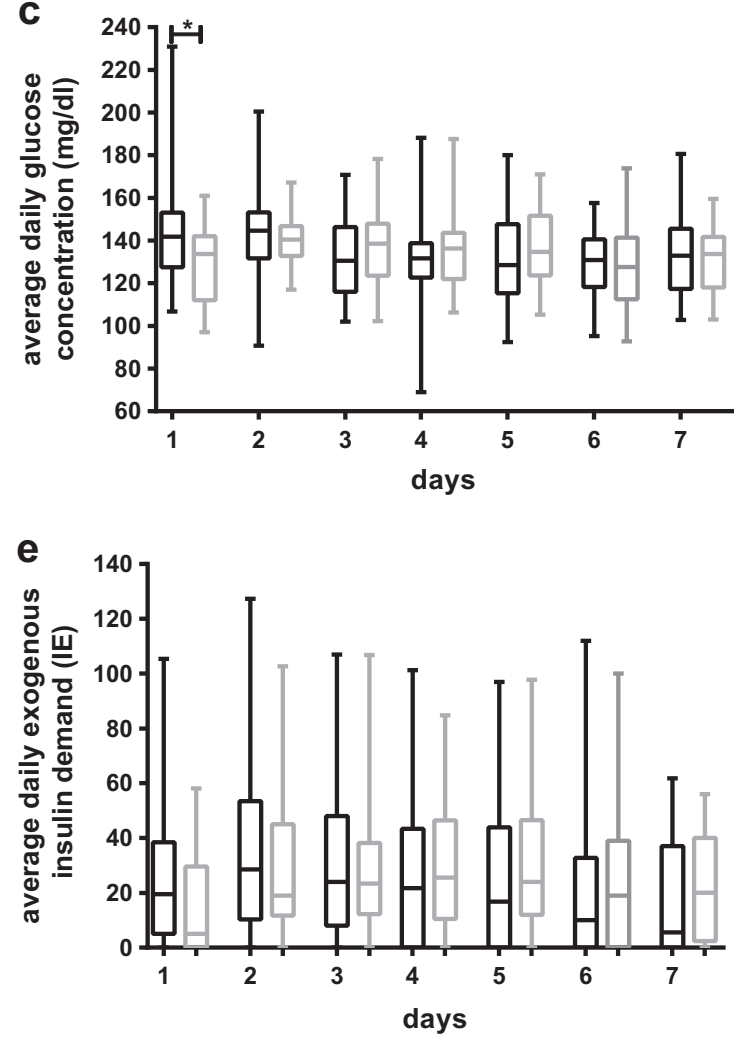

Fig. 2 Average a percentage of nutrition targeted by administered enteral nutrition per $24 \mathrm{~h}$, b daily total calories, c daily glucose concentrations, $\mathbf{d}$ glucose variability and exogenous insulin demand per b

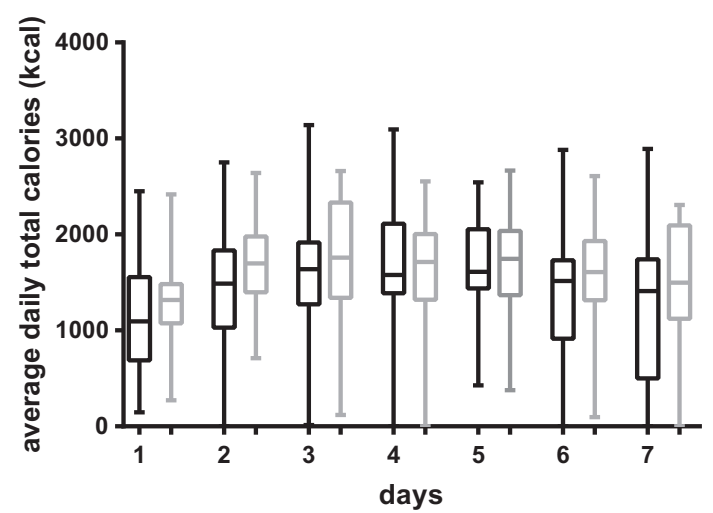

d

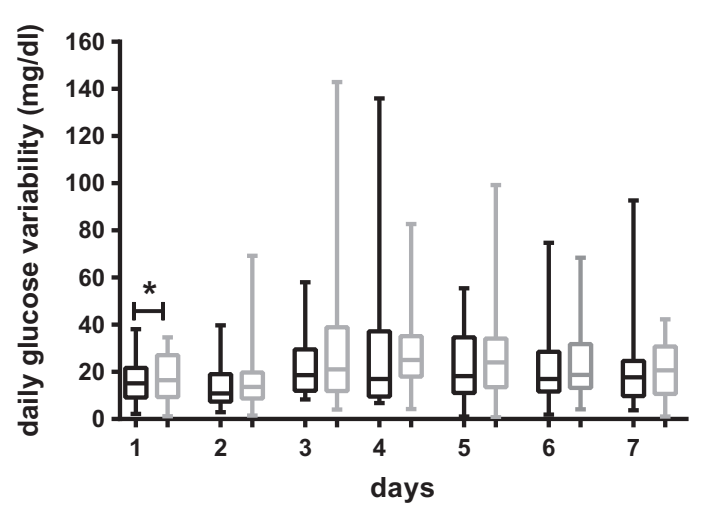

$24 \mathrm{~h}$ over the duration of the study. $*<0.05$. Black-fat-based enteral nutrition (group A). Gray-glucose-based enteral nutrition (group B)

\section{Nutrition Related Side Effects}

Patients had similar rates of hypertriglyceridemia over the study period (group A: $n=5$ vs group B: $n=1$ events, $p=0.215$ ). Cholestasis was also similar between groups with $n=6$ patients in group A and $n=4$ patients in group B $(p=0.731)$. Patients in both groups had similar rates of clinically significant GRV (group A: $n=19$ vs group B: $n=18$ events, $p=1.0$ ), diarrhea (group A: $n=22$ vs group B: $n=26, p=0.646$ ), and vomiting (group A: $n=$ 2 vs group $\mathrm{B} n=3, p=1.0$ ). The use of metoclopramide was similar in both groups $(p=0.114)$, whereas patients who received fat-based EN trended to receive more erythromycin throughout the study period $(p=0.055)$. However, patient days on erythromycin did not differ between groups (group A: 7 days, group B: 2 days, $p=$ 0.174). Overall, prokinetics use was similar between groups $(p=0.276)$. 


\section{Discussion}

In this prospective, randomized, clinical cohort study we found that administration of either fat-based or glucosebased EN caused no significant differences in various measures of glucose homeostasis in medical critically ill patients. While baseline REE was similar between groups, we showed that EE significantly increased over the study duration of 7 days only in patients receiving fatbased EN.

In critically ill patients stress hyperglycemia is commonly observed and results from a complex interplay of catecholamines, counter-regulatory hormones as well as proinflammatory cytokines, causing an aggravation of insulin resistance and dysglycemia. These derangements in carbohydrate metabolism have been shown to be related to outcome and the severity of illness [21]. Latter finding was confirmed by our study, since we found FPG to be elevated at baseline in both study groups. Additionally, severity of illness was profound, as reflected by high severity scores of our patients.

Stress hyperglycemia could be aggravated by an additional exogenous glucose administration, as performed during nutritional support. In the present study, the amount of exogenous glucose administration via EN differed significantly between groups. Patients who were fed a fatbased diet received $51 \%$ less glucose than patients on a glucose-based diet. However, the lower glucose supply did not result in differences of the average daily serum glucose concentration, of glucose AUC or daily glucose variability during the entire study period. In critically ill patients blood glucose concentrations should be monitored carefully and can be adjusted within a recommended range by a continuous insulin administration. In our study, the insulin dosage required to maintain the blood glucose concentration below $150 \mathrm{mg} / \mathrm{dl}$ did not differ between groups. These findings indicate that a fat-based EN, despite a considerably lower glucose administration, cannot ameliorate dysglycemia in critically ill medical patients. Nonetheless, the unaffected dysglycemia could also be an expression of diminished insulin sensitivity in critically ill medical patients receiving a fat-based formula. In critically ill medical patients insulin sensitivity has been shown to be reduced by $70 \%$ compared to healthy controls [7]. This impairment might be aggravated by the higher fat administration, since high free fatty acid levels could further worsen insulin sensitivity [6]. However, we did not measure insulin sensitivity or free fatty acids concentration.

Our findings of comparable glucose homeostasis are in contrast to Huschak et al. [9] who found lower blood glucose concentrations during a lipid-based nutrition compared to a glucose-based nutrition. However, in this study artificial nutrition was delivered to trauma patients and mainly parenterally. Moreover, the composition of administered fat emulsions differed substantially using either an emulsion based on olive oil or a soybean derived emulsion. This study is in accordance with Tappy et al. [8] who also found a lower plasma glucose during a lipid-based total parenteral nutrition in surgical patients. However, the different findings of these two studies compared to our study could be explained by the enteral route of substrate administration. Although the enteral route is preferable in critically ill patients, it remains unclear whether macronutrients are satisfactorily absorbed due to a possible exocrine pancreatic insufficiency and/or alterations in the intestinal mucosa [22]. In addition, an increase of the hyperglycemic risk seems to be less important during EN than during parenteral nutrition, which has been attributed to the incretin effect [23].

Guidelines recommend to assess energy demands of critically ill patients by indirect calorimetry in order to avoid overfeeding as well as underfeeding [2]. In the present study this measurement revealed no differences in REE between both groups after an overnight fast directly before EN was started. REE was found to be in the range published for critically ill medical patients previously [13]. The feeding of a glucose-based EN caused no significant change of energy expenditure during the entire study period. However, in critically ill medical patients receiving fat-based EN, energy expenditure increased constantly over time from day 1 to day 7 by $18.5 \%$. The increase of energy expenditure could reveal a considerable diet-induced thermogenesis in our patients receiving a fat-based EN, which is part of the total energy expenditure. This increase seemed not to be caused by changes in substrate oxidation rates, since we found no differences in RQ comparing both groups neither at baseline nor on days 3 or 7 . RQ was found to be low at baseline indicating that fat was the main substrate oxidized after an overnight fast. During EN, while keeping the energy intake constant, the RQ in our study subjects rose, but never exceeded 1.0, pointing out that no net de novo lipogenesis was present (i.e. total lipid oxidation was constantly higher than total lipid synthesis). However, indirect calorimetry only records the net result of metabolic processes [24]. Thus, it seems feasible that the varying composition of the two EN influenced metabolic pathways in a different manner in our critically ill medical patients. Another possibility for the increase of the energy expenditure during fat-based EN might be an induction of uncoupling proteins. These proteins, located in the mitochondrial inner membrane, dissipate energy present in the proton-motive force as heat and can be activated by free fatty acids [25]. Taken together, it seems unnecessary to use fatbased EN formulas in the early period of ICU stay, as it has no beneficial effect on glucose homeostasis. In contrast, the 
increase in energy expenditure due to diet-induced thermogenesis might induce or even aggravate underfeeding.

A limitation of our study is that insulin resistance was not measured using the hyperinsulinemic clamp technique, which is considered the gold standard [26] and that the investigators were not blinded with regard to the nutrition formula. We also did not directly determine intestinal glucose absorption, which might be compromised due to delayed gastric emptying, intestinal edema, reduction of enzymes in the brush border, and reduced splanchnic perfusion [27, 28]. However, these potential alterations in nutrient absorption can be expected to be comparable in the patients of both study groups. Furthermore, the fiber content of the two nutrition formulas differed. This might have influenced the intestinal microbiota differently leading to alterations in glucose homeostasis. Yet, it remains to be determined whether these small differences in fiber content are clinically relevant or can impact the intestinal microbiota within one week.

\section{Conclusion}

In summary, fat-based and glucose-based EN influence glucose homeostasis and insulin demand similarly, yet dietinduced thermogenesis was substantially higher in critically ill patients receiving fat-based enteral nutrition.

Funding This study was supported by a scientific grant of Fresenius Kabi, Austria.

\section{Compliance with Ethical Standards}

Conflict of interest The authors declare that they have no conflict of interest.

\section{References}

1. Kreymann KG, Berger MM, Deutz NE, Hiesmayr M, Jolliet P, Kazandjiev $\mathrm{G}$, et al. ESPEN guidelines on enteral nutrition: intensive care. Clin Nutr. 2006;25:210-23. https://doi.org/10. 1016/j.clnu.2006.01.021

2. Singer P, Berger MM, Van den Berghe G, Biolo G, Calder P, Forbes A, et al. ESPEN guidelines on parenteral nutrition: intensive care. Clin Nutr. 2009;28:387-400. https://doi.org/10.1016/j. clnu.2009.04.024

3. Faisy C, Lerolle N, Dachraoui F, Savard JF, Abboud I, Tadie JM, et al. Impact of energy deficit calculated by a predictive method on outcome in medical patients requiring prolonged acute mechanical ventilation. Br J Nutr. 2009;101:1079-87. https://doi.org/10.1017/ S0007114508055669

4. Singer P, Anbar R, Cohen J, Shapiro H, Shalita-Chesner M, Lev $\mathrm{S}$, et al. The tight calorie control study (TICACOS): a prospective, randomized, controlled pilot study of nutritional support in critically ill patients. Intensive Care Med. 2011;37:601-9. https://doi. org/10.1007/s00134-011-2146-z
5. Anbar R. Enteral nutrition. World Rev Nutr Diet. 2013;105:50-58. https://doi.org/10.1159/000341267

6. Dungan KM, Braithwaite SS, Preiser JC. Stress hyperglycemia. Lancet. 2009;373:1798-807.

7. Zauner A, Nimmerrichter P, Anderwald C, Bischof M, Schiefermeier M, Ratheiser K, et al. Severity of insulin resistance in critically ill medical patients. Metabolism. 2007;56:1-5.

8. Tappy L, Schwarz JM, Schneiter P, Cayeux C, Revelly JP, Fagerquist CK, et al. Effects of isoenergetic glucose-based or lipid-based parenteral nutrition on glucose metabolism, de novo lipogenesis, and respiratory gas exchanges in critically ill patients. Crit Care Med. 1998;26:860-7.

9. Huschak G, Zur Nieden K, Hoell T, Riemann D, Mast H, Stuttmann R. Olive oil based nutrition in multiple trauma patients: a pilot study. Intensive Care Med. 2005;31:1208-1208.

10. Le Gall JR, Lemeshow S, Saulnier F. A new Simplified Acute Physiology Score (SAPS II) based on a European/North American multicenter study. JAMA. 1993;270:2957-63.

11. Vincent JL, Ferreira F, Moreno R. Scoring systems for assessing organ dysfunction and survival. Crit Care Clin. 2000;16:353-66.

12. Elke G, Felbinger TW, Heyland DK. Gastric residual volume in critically ill patients: a dead marker or still alive? Nutr Clin Pract. 2015;30:59-71. https://doi.org/10.1177/0884533614562841

13. Zauner C, Schuster BI, Schneeweiss B. Similar metabolic responses to standardized total parenteral nutrition of septic and nonseptic critically ill patients. Am J Clin Nutr. 2001;74:265-70.

14. Wooley JA. Indirect calorimetry: applications in practice. Respir Care Clin N Am. 2006;12:619-33. https://doi.org/10.1016/j.rcc. 2006.09.005

15. Tissot S, Delafosse B, Bertrand O, Bouffard Y, Viale JP, Annat G. Clinical validation of the Deltatrac monitoring system in mechanically ventilated patients. Intensive Care Med. 1995;21:149-53.

16. Nunn J. Applied respiratory physiology. London: Butterworth; 1977.

17. Ferrannini E. The theoretical bases of indirect calorimetry: a review. Metabolism. 1988;37:287-301.

18. Weir JB. New methods for calculating metabolic rate with special reference to protein metabolism. J Physiol. 1949;109:1-9.

19. Livesey G, Elia M. Estimation of energy expenditure, net carbohydrate utilization, and net fat oxidation and synthesis by indirect calorimetry: evaluation of errors with special reference to the detailed composition of fuels. Am $J$ Clin Nutr. 1988;47:608-28.

20. Egi M, Bellomo R, Stachowski E, French CJ, Hart G. Variability of blood glucose concentration and short-term mortality in critically ill patients. Anesthesiology. 2006;105:244-52.

21. Preiser JC, Ichai $\mathrm{C}$, Orban JC, Groeneveld AB. Metabolic response to the stress of critical illness. $\mathrm{Br} \mathrm{J}$ Anaesth. 2014;113:945-54. https://doi.org/10.1093/bja/aeu187

22. Wang S, Ma L, Zhuang Y, Jiang B, Zhang X. Screening and risk factors of exocrine pancreatic insufficiency in critically ill adult patients receiving enteral nutrition. Crit Care. 2013;17:R171 https://doi.org/10.1186/cc12850

23. Drucker DJ. The biology of incretin hormones. Cell Metab. 2006;3:153-65. https://doi.org/10.1016/j.cmet.2006.01.004

24. Carlson GL. Nutrient induced thermogenesis. Baillieres Clin Endocrinol Metab. 1997;11:603-15.

25. Rousset S, Alves-Guerra MC, Mozo J, Miroux B, CassardDoulcier AM, Bouillaud F, et al. The biology of mitochondrial uncoupling proteins. Diabetes. 2004;53 Suppl 1:S130-135.

26. DeFronzo RA, Tripathy D. Skeletal muscle insulin resistance is the primary defect in type 2 diabetes. Diabetes Care. 2009;32 Suppl 2:S157-163. https://doi.org/10.2337/dc09-S302

27. Chapman MJ, Fraser RJ, Matthews G, Russo A, Bellon M, Besanko LK, et al. Glucose absorption and gastric emptying in 
critical illness. Crit Care. 2009;13:R140 https://doi.org/10.1186/ cc8021

28. Burgstad CM, Besanko LK, Deane AM, Nguyen NQ, SaadatGilani K, Davidson G, et al. Sucrose malabsorption and impaired mucosal integrity in enterally fed critically ill patients: a prospective cohort observational study. Crit Care Med. 2013;41:1221-8. https://doi.org/10.1097/CCM.0b013e31827ca $2 \mathrm{fa}$ 